\title{
Nutrition, Vitamin E
}

National Cancer Institute

\section{Source}

National Cancer Institute. Nutrition, Vitamin E. NCI Thesaurus. Code C15929.

Role of Vitamin E (alpha-tocopherol), its precursons, and its analogs in cancer causation or prevention and in general health. 